\title{
Identification of Adult Fasciola spp. Using Matrix-Assisted Laser/Desorption Ionization Time-of-Flight (MALDI-TOF) Mass Spectrometry
}

\author{
Issa Sy ${ }^{1}{ }^{(0}$, Lena Margardt ${ }^{1}$, Emmanuel O. Ngbede ${ }^{1,2}{ }^{\circledR}$, Mohammed I. Adah $^{2}$, Saheed T. Yusuf ${ }^{3}$, \\ Jennifer Keiser ${ }^{4,5}{ }^{\circledR}$, Jacqueline Rehner ${ }^{1}$, Jürg Utzinger ${ }^{4,5}$, Sven Poppert ${ }^{4,5}$ and Sören L. Becker ${ }^{1,4,5, *(\mathbb{D})}$
}

1 Institute of Medical Microbiology and Hygiene, Saarland University, 66421 Homburg/Saar, Germany; issa.sy7@hotmail.com (I.S.); lena.margardt@uks.eu (L.M.); drngbede@hotmail.com (E.O.N.); jacqueline.rehner@uks.eu (J.R.)

2 College of Veterinary Medicine, Federal University of Agriculture, Makurdi 970213, Nigeria; adahmi@yahoo.com

3 Kubwa Abattoir, Public Health Section, Department of Veterinary Services, Federal Capital Territory Administration, Abuja 901101, Nigeria; saheed.ty@gmail.com

4 Swiss Tropical and Public Health Institute, CH-4002 Basel, Switzerland; jennifer.keiser@swisstph.ch (J.K.); juerg.utzinger@swisstph.ch (J.U.); sven.poppert@swisstph.ch (S.P.)

5 University of Basel, $\mathrm{CH}-4003$ Basel, Switzerland

* Correspondence: soeren.becker@uks.eu; Tel.: +49-6841-16-23901

\section{check for}

updates

Citation: Sy, I.; Margardt, L.; Ngbede, E.O.; Adah, M.I.; Yusuf, S.T.; Keiser, J.; Rehner, J.; Utzinger, J.; Poppert, S.; Becker, S.L. Identification of Adult Fasciola spp. Using Matrix-Assisted Laser/Desorption Ionization Time-of-Flight (MALDI-TOF) Mass Spectrometry. Microorganisms 2021, 9 , 82. https://doi.org/10.3390/ microorganisms 9010082

Received: 18 November 2020 Accepted: 25 December 2020 Published: 31 December 2020

Publisher's Note: MDPI stays neutral with regard to jurisdictional clai$\mathrm{ms}$ in published maps and institutional affiliations.

Copyright: (C) 2020 by the authors. Licensee MDPI, Basel, Switzerland. This article is an open access article distributed under the terms and conditions of the Creative Commons Attribution (CC BY) license (https:// creativecommons.org/licenses/by/ $4.0 /)$.

\begin{abstract}
Fascioliasis is a neglected trematode infection caused by Fasciola gigantica and Fasciola hepatica. Routine diagnosis of fascioliasis relies on macroscopic identification of adult worms in liver tissue of slaughtered animals, and microscopic detection of eggs in fecal samples of animals and humans. However, the diagnostic accuracy of morphological techniques and stool microscopy is low. Molecular diagnostics (e.g., polymerase chain reaction (PCR)) are more reliable, but these techniques are not routinely available in clinical microbiology laboratories. Matrix-assisted laser/desorption ionization time-of-flight (MALDI-TOF) mass spectrometry (MS) is a widely-used technique for identification of bacteria and fungi; yet, standardized protocols and databases for parasite detection need to be developed. The purpose of this study was to develop and validate an in-house database for Fasciola species-specific identification. To achieve this goal, the posterior parts of seven adult $F$. gigantica and one adult $F$. hepatica were processed and subjected to MALDI-TOF MS to create main spectra profiles (MSPs). Repeatability and reproducibility tests were performed to develop the database. A principal component analysis revealed significant differences between the spectra of F. gigantica and F. hepatica. Subsequently, 78 Fasciola samples were analyzed by MALDI-TOF MS using the previously developed database, out of which $98.7 \%(n=74)$ and $100 \%(n=3)$ were correctly identified as F. gigantica and F. hepatica, respectively. Log score values ranged between 1.73 and 2.23, thus indicating a reliable identification. We conclude that MALDI-TOF MS can provide species-specific identification of medically relevant liver flukes.
\end{abstract}

Keywords: diagnosis; Fasciola gigantica; Fasciola hepatica; fascioliasis; helminth; matrix-assisted laser/desorption ionization time-of-flight (MALDI-TOF) mass spectrometry; trematode

\section{Introduction}

Fascioliasis is a food-borne parasitic disease in animals and humans, caused by the digenetic trematodes Fasciola gigantica and Fasciola hepatica [1]. While the occurrence of F. gigantica is restricted to areas of Asia and Africa [2,3], F. hepatica is widely distributed throughout Africa, Asia, Europe, Oceania and the Americas [4-7]. Fascioliasis and other food-borne trematode infections are classified as neglected tropical diseases. An estimated 2.4-17 million people are infected with Fasciola spp. [8]. However, these numbers may be considerable underestimations of the true number of infections, and hence, the global 
disease burden. In veterinary surveys, high prevalences of fascioliasis have been reported. For example, recent studies carried out in Lake Chad area and in a coastal region of Vietnam reported a prevalence in cattle of $68 \%$ and $23 \%$, respectively; additionally, a significant risk for spillover of infections from animals to humans was noted $[9,10]$.

The two Fasciola species share similar life cycles [1,11]. In brief, the life cycles are complex and require a snail intermediate host of the family Lymnaeidae (e.g., Galba truncatula for F. hepatica, Lymnaea natalensis for F. gigantica [12,13], and Pseudosuccinea columella for both species [14]). Adult worms are located in the biliary ducts of the definitive host (e.g., ruminants and humans). They release unembryonated eggs that are passed with the feces. These eggs become embryonated by contact with unprotected surface water. Subsequently, a miracidium hatches, which in turn infects the intermediate snail host. Free-swimming cercariae are released from the snail that form metacercariae and can attach to aquatic vegetation. Human infection occurs most frequently by the unintended ingestion of freshwater or vegetables (e.g., watercress) that are contaminated with infective metacercariae [7]. Following ingestion by humans or animals, immature worms excyst in the duodenum and penetrate the intestinal wall from where they migrate through the liver parenchyma [15].

Current methods used for the diagnosis of human and veterinary fascioliasis, in particular for the identification of adult worms, rely on morphological analyses of the trematodes [16], molecular methods (e.g., polymerase chain reaction (PCR)) and sequencing $[7,11,17]$. The latter two approaches have several limitations, including a lack of rigorous standardization of the morphological identification in different settings, relatively high costs and unavailability of PCR-based testing using specific primers for e.g., trematodes outside highly specialized research laboratories. While the morphological identification is a rapid and less costly procedure, Fasciola spp. are rarely detected outside endemic settings and the waning of parasitological experience among laboratory technicians in clinical laboratories is a particular challenge. Hence, the development of an accurate, rapid, less expensive and more accessible diagnostic technique for parasite identification would be desirable. During the past decade, matrix-assisted laser desorption/ionization time-of-flight (MALDI-TOF) mass spectrometry (MS) has become a widely employed tool for the diagnosis of bacteria and fungi in clinical samples [18-20]. More recently, a variety of studies have reported the use of MALDI-TOF MS as a potentially promising tool for the identification of mosquitoes [21,22], ticks [23], and, to a lesser extent, parasites (protozoa and helminths) [24]. In contrast to PCR-based techniques, MALDI-TOF MS allows for a broad, "untargeted" detection of microorganisms if these are present in the database used for pathogen identification. The goal of this study was to validate MALDI-TOF MS for the identification and differentiation of adult Fasciola.

\section{Materials and Methods}

\subsection{Ethics Statement}

For adult Fasciola samples originating from Nigeria, written permission was obtained from the management board of the Kubwa abattoir (Abuja, Nigeria) for collection and subsequent analyses of the fluke samples from slaughtered cattle (reference no.: FCT/ARD/TRN/004, 17 October 2019). Adult F. hepatica specimens were obtained from livers from a slaughterhouse in central Switzerland. Note that such specimens are utilized for routine diagnostic work and research purposes at the Swiss Tropical and Public Health Institute (Swiss TPH; Basel, Switzerland), adhering to local laws and regulations.

\subsection{Sample Collection}

For the current investigation, adult Fasciola worms were collected by experienced veterinarians from the liver tissue of slaughtered cattle at the Kubwa abattoir in Abuja, the Federal Capital Territory of Nigeria in December 2019 and January 2020. Samples were stored in $70 \%(v / v)$ ethanol at room temperature and were transferred to the Institute of Medical Microbiology and Hygiene in Homburg, Germany. 
Additional samples were collected in early 2020 from the livers of cattle from a slaughterhouse in Oensingen, Switzerland. Livers were routinely screened by the slaughterhouse veterinarians. Infected livers were put aside and, on the same day, transferred and examined by experienced laboratory technicians at Swiss TPH. Fasciola specimens were stored in $0.7 \%(v / v)$ sodium chloride solution $(\mathrm{NaCl})$ and transferred on ice to the Institute of Medical Microbiology and Hygiene in Homburg. Upon receipt in Homburg, samples were kept at $-20{ }^{\circ} \mathrm{C}$ pending further examination.

\subsection{Sample Preparation}

Adult worms were removed from the storage solution and dried at room temperature to allow for evaporation of organic solvents. Two small pieces (each weighing approximately $15 \mathrm{mg}$ ) of the posterior part of each fluke were cut with a sterile scalpel to be used for subsequent molecular analyses and MALDI-TOF MS.

\subsection{Molecular Analysis}

\subsubsection{DNA Extraction, PCR and Sequencing}

The DNeasy Blood and Tissue Kit (Qiagen GmbH; Hilden, Germany) was utilized for DNA extraction, adhering to the manufacturer's instructions. Briefly, pieces measuring approximately $5 \mathrm{~mm}$ of each fluke were placed into a $1.5 \mathrm{~mL}$ Eppendorf tube, adding $280 \mu \mathrm{L}$ lysis buffer and $20 \mu \mathrm{L}$ proteinase $\mathrm{K}$. The flukes were gently crushed in this mixture. Next, the mixture was incubated using a thermomixer (Eppendorf; Hamburg, Germany) at $56{ }^{\circ} \mathrm{C}$ and $800 \times g$ for $1 \mathrm{~h}$. After digestion, a washing step was performed using AW1 and AW2 buffers from the Qiagen kits and a column with a silica membrane. The extracted DNA was eluted in $200 \mu \mathrm{L}$ of AE buffer (Qiagen; Hilden, Germany) and stored at $-20^{\circ} \mathrm{C}$ pending molecular analyses.

PCR amplification of the partial mitochondrial cytochrome oxidase 1 gene (COX1) of all Fasciola specimens was carried out in Homburg, using a previously described protocol with the forward primer $5^{\prime}$-TTGGTTTTTTGGGCATCCT- $3^{\prime}$ and the reverse primer $5^{\prime}$-AGGCCACCACCAAATAAAAGA- $3^{\prime}$ [6]. The amplicons generated were sequenced using the Capillary Electrophoretic GenomeLab genetic analysis system (Beckman Coulter; Brea, CA, USA).

\subsubsection{Sequence Analysis and Species Identification}

The forward and reverse sequences obtained were edited and combined to generate a consensus sequence for each specimen, using the software BioEdit ${ }^{\odot}$ version 7.2.5 (Tom Hall; Carlsbad, CA, USA) [25]. The consensus sequences were then queried against the National Center for Biotechnology Information (NCBI) GenBank database for identification, using the Basic Local Alignment Search Tool (BLASTn) [26]. Sequences obtained from the eight isolates that were used for MALDI-TOF MS database development during this study were submitted to GenBank, comprising the consecutive accession numbers MW258701 to MW258708.

\subsection{MALDI-TOF Analysis}

\subsubsection{Protein Extraction}

A small piece corresponding to approximately $15 \mathrm{mg}$ from the posterior part of each adult fluke was cut thinly with a sterile scalpel in order to facilitate the release of molecules from other locations than the fluke's surface area, and was subsequently put into a $1.5 \mathrm{~mL}$ tube (Figure 1). Of note, no eggs can be found in the posterior part of Fasciola, as uterine structures are localized in the anterior part of this helminth. The "complete extraction protocol", recommended by the manufacturer (Bruker Daltonics; Bremen, Germany) for protein extraction from bacteria for subsequent MALDI-TOF analysis, was readily adapted to the fluke samples. In brief, $300 \mu \mathrm{L}$ of LC-MS grade water (Merck KG; Darmstadt, Germany) and $900 \mu \mathrm{L}$ of $100 \%(v / v)$ ethanol (Merck KG) were added to the samples and mixed by vortexing. The mixture was centrifuged at $18,312 \times g$ for $2 \mathrm{~min}$ and the 
supernatant discarded. The pellet was resuspended in $50 \mu \mathrm{L}$ of $70 \%$ formic acid and $50 \mu \mathrm{L}$ of acetonitrile and mixed by vortexing. Of note, we also employed additional steps, including the use of Zirconium beads, during the development of our helminth extraction protocol, but did not observe differences in the obtained spectral profile. Hence, we decided not to use beads to reduce the working steps and the hands-on time per sample.

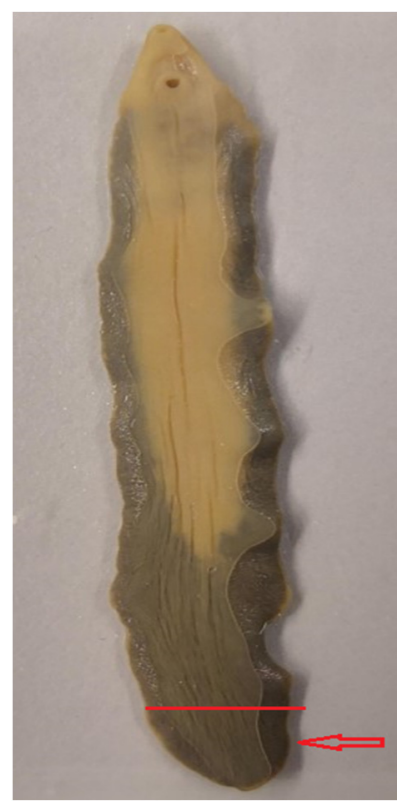

Figure 1. Morphology of an adult Fasciola gigantica fluke. The red line shows the area where the incision of the helminth was performed, and the red arrow indicates the posterior body part that was used for molecular analysis and MALDI-TOF MS.

\subsubsection{Target Plate Preparation and Measurements}

The protein extracts obtained with the mix of formic acid and acetonitrile above was centrifuged at $18,312 \times g$ for $2 \mathrm{~min}$ and $1 \mu \mathrm{L}$ of the clear supernatant spotted onto the MALDI-TOF target plate, followed by overlaying with $1 \mu \mathrm{L}$ of $\alpha$-cyano-4-hydroxycinnamic acid (CHCA) matrix solution (Bruker Daltonics), composed of saturated CHCA 50\% $(v / v)$ of acetonitrile, $2.5 \%(v / v)$ of trifluoroacetic acid and $47.5 \%(v / v)$ of LC-MS grade water. Bacterial test standard (BTS) (Bruker Daltonics), which is an extract of Escherichia coli that is spiked with two high molecular weight proteins, was used to calibrate the machine. After drying at room temperature, the plate was placed into the Microflex LT Mass Spectrometer (Bruker Daltonics) for MALDI-TOF MS.

\subsubsection{MALDI-TOF MS Parameters}

Measurements were performed using the AutoXecute algorithm in the FlexControl ${ }^{\circledR}$ software version 3.4 (Bruker Daltonics). For each spot, 240 laser shots in six random positions were carried out automatically to generate protein mass profiles in linear positive ion mode with a laser frequency of $60 \mathrm{~Hz}$, a high voltage of $20 \mathrm{kV}$ and a pulsed ion extraction of $180 \mathrm{~ns}$. Mass charge ratios range $(m / z)$ were measured between $2 \mathrm{k}$ and $20 \mathrm{k}$ Da.

\subsubsection{Spectral Analysis and Database Creation}

For the creation of species-specific main spectra profiles (MSPs), protein extracts of one F. hepatica and seven F. gigantica specimens were spotted onto the MALDI-TOF target plate eight times per sample. Next, each spot was measured four times to generate 32 raw spectra per sample. For each specimen, this procedure was carried out on two replicates on the same day (to demonstrate repeatability) and on one replicate on a different day (to demonstrate reproducibility). Hence, a total of 96 raw spectra were acquired for each sample, using the FlexControl ${ }^{\circledR}$ software version 3.4 (Bruker Daltonics). These raw spectra 
were analyzed and curated using the FlexAnalysis ${ }^{\circledR}$ software version 3.4 (Bruker Daltonics) for a "cleaning step", i.e., to withdraw all flatlines and outlier peaks and to smoothen intensities and edit peak shifts within spectra whenever these exceeded 500 ppm. Following this editing step, replicates containing at least 22 remaining spectra were randomly chosen for the creation of species-specific MSPs. These MSPs were created using the automatic function of the MALDI Biotyper Compass Explorer ${ }^{\circledR}$ software version 3.0 (Bruker Daltonics). Finally, the newly created MSPs of both Fasciola species were included in a previously developed in-house MALDI-TOF database for helminth identification, which already contained several nematodes (e.g., Ascaris lumbricoides), cestodes (e.g., Taenia saginata) and trematodes (e.g., Schistosoma mansoni).

MSP dendrogram analysis was carried out with the MALDI Biotyper Compass Explorer and employed the following parameters: distance correlation, linkage by average and score threshold values of 300 and 0 (arbitrary unit) for a single and a related organism, respectively. For a principal component analysis and a discriminatory analysis of the species-specific MSPs, we used the software BioNumerics ${ }^{\circledR}$ version 7.5 (Applied Maths N.V.; Sint-Martens-Latem, Belgium).

\subsubsection{Validation Test}

The newly developed in-house database was subjected to two different validation procedures. First, an internal validation, during which all raw spectra of Fasciola spp. obtained during the process of MSP creation were analyzed. Second, an external validation, during which raw spectra from new, unidentified Fasciola specimens were measured by MALDI-TOF MS to assess the database's ability to reliably identify these samples.

All spectra were tested against the commercially available official database released by Bruker Daltonics for identification of bacteria and fungi (Bruker Taxonomy, Maldi Biotyper Compass Explorer version 3.0). Next, the spectra were subjected to a combination of the official database and our in-house helminth database. The reliability of identification was assessed by log score values (LSVs), which are generated for each result. We adhered to the "official" grading system put forth by the manufacturer for bacteria (i.e., LSVs may range between 0 and 3; LSV of $\geq 1.70$ is considered as a threshold for identification; LSVs ranging between 1.70 and 1.99 indicate a reliable identification at the genus level; and LSVs equal to or higher than 2.0 are interpreted as reliable species identification).

\section{Results}

\subsection{Comparative Analysis of Samples Used for MSP Database Creation}

For the creation of MSPs to be included in the in-house database, we included seven randomly selected adult flukes obtained from cattle in Nigeria, which had previously been identified as F. gigantica based on morphological characteristics, and one adult F. hepatica provided from Switzerland. Partial sequencing of the COX1 confirmed the species diagnosis in the eight reference samples. In comparison to previously deposited sequences, the BLAST analysis revealed sequence homologies between $98.5 \%$ and $99.8 \%$ for F. gigantica (reference accession numbers: MN586868.1, MN586869.1, MN913872.1 and MN913873.1) and $99.4 \%$ for F. hepatica (reference accession number: GQ231551.1). The visualization of spectra obtained by MALDI-TOF MS is displayed in Figure 1. Considerable differences between F. gigantica and F. hepatica were observed (Figure 2A), which were confirmed by a dendrogram analysis (Figure 2B). Of note, there was some heterogeneity with regard to the spectra within the F. gigantica cluster (e.g., samples FGN23 and FGN24), which was further substantiated by a principal component analysis and discriminant analysis (Figure 3 ). The COX1 sequences of these two samples (FGN23 and FGN24) showed sequence homologies of $99.5 \%$ and $98.6 \%$, respectively, to the GenBank reference sequences of F. gigantica. 


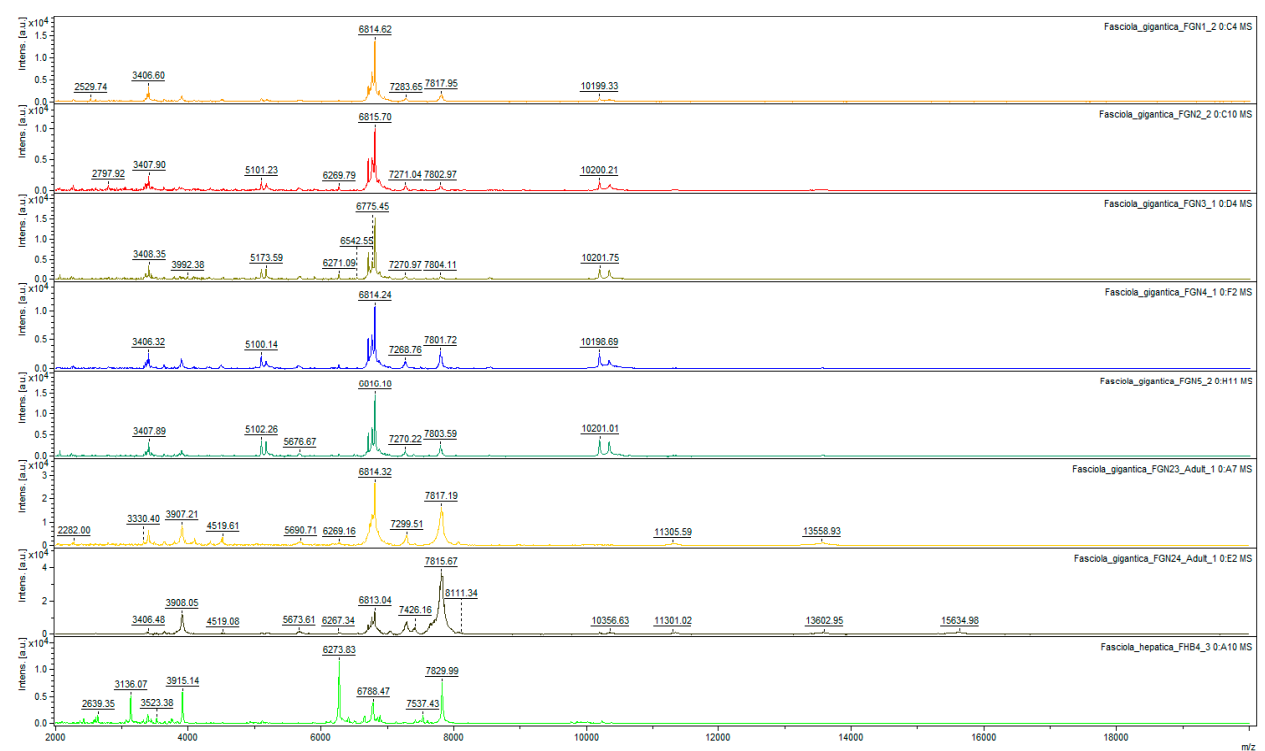

(A)

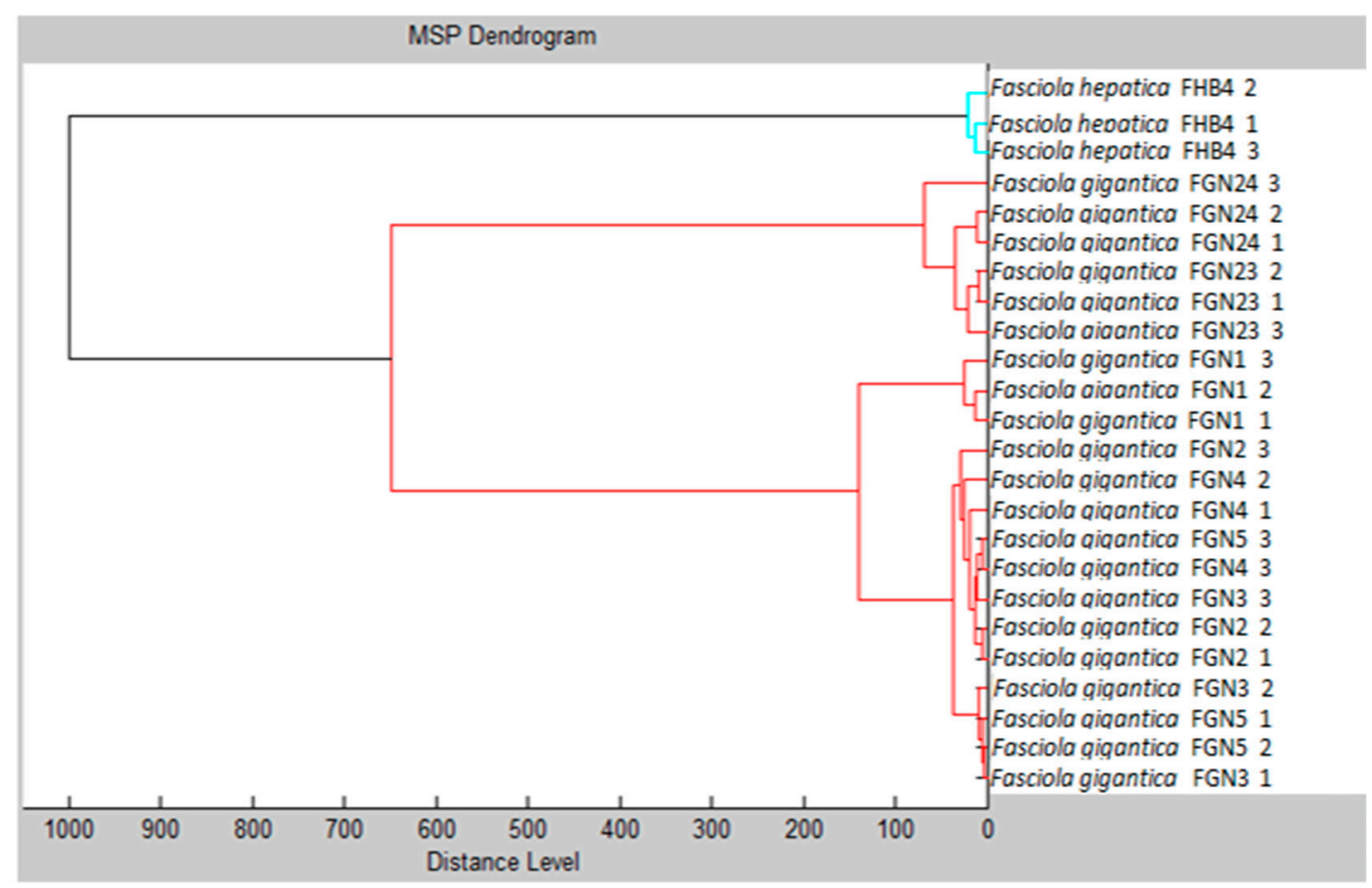

(B)

Figure 2. (A) Spectra obtained by matrix-assisted laser/desorption ionization time-of-flight (MALDI-TOF) mass spectrometry (MS) for seven adult Fasciola gigantica (FG) specimens and one adult Fasciola hepatica (FH) specimen. The peak intensities of ionised molecules are shown on the y-axis and the corresponding mass on the $x$-axis. Numbers indicate the resulting mass-to-charge-ratios. (B) Dendrogram analysis displaying the relatedness of the different samples. Of note, each specimen was measured thrice to assure reproducibility. 
A

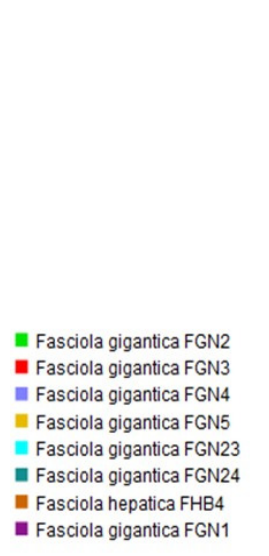

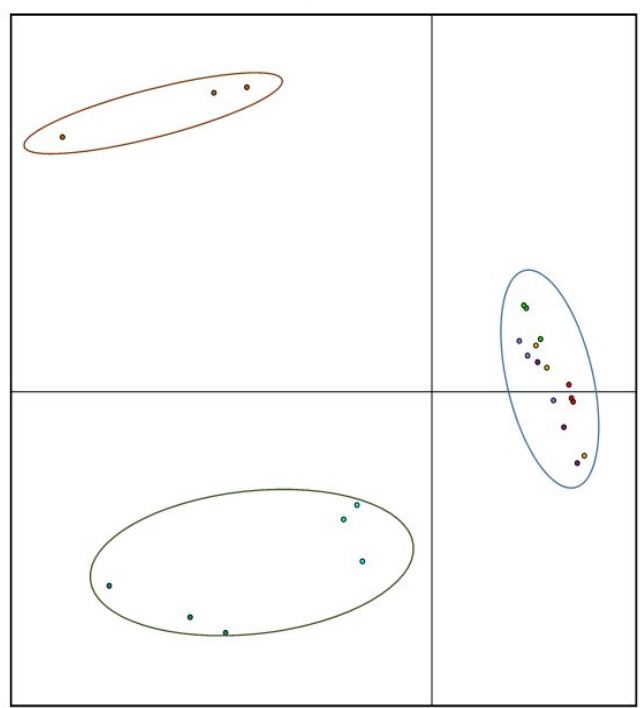

Figure 3. Statistical analysis of main spectra profiles (MSPs) obtained during the creation of an in-house database for identification of adult Fasciola gigantica (FG) and Fasciola hepatica (FH) using MALDI-TOF MS. (A) Three-dimensional view of a principal component analysis, displaying three distinct clusters, i.e., (i) F. hepatica FHB4; (ii) F. gigantica FGN23 and FGN24; and (iii) the remaining F. gigantica samples. (B) Two-dimensional view of a discriminant analysis, using the same samples. The analyses were carried out using the software BioNumerics.

\subsection{Internal Database Validation}

When the reference spectra were analyzed with the commercially available database for bacteria and fungi identification, no reliable identification was achieved, and all LSVs were below 1.7. In the subsequent analysis of the raw spectra obtained during MSP creation, which used a combination of the official and the in-house database, a reliable species identification as either F. gigantica or F. hepatica was achieved in all eight samples, with LSVs ranging from 2.17 to 2.86 (average LSV $=2.5$ ).

\subsection{Analysis of Samples for External Database Validation}

A total of 78 Fasciola samples were subjected to MALDI-TOF analysis, using the newly developed database. There were 75 samples from Nigeria that had previously been identified as F. gigantica, based on morphological characteristics. The remaining three specimens stemmed from Switzerland and were reported as F. hepatica. PCR was performed on all samples and confirmed the species identification in all cases.

When using the previously developed in-house database and considering an LSV threshold of $\geq 1.70$, MALDI-TOF MS correctly identified 74/75 (98.7\%) of the F. gigantica and $3 / 3(100 \%)$ of the F. hepatica samples. In one sample, MALDI-TOF MS did not identify sufficient protein spectra to provide an identification. When an LSV threshold of $\geq 2.0$ was used, the identification rate was $31 / 75$ (41.3\%) for F. gigantica, while it remained unchanged for F. hepatica (Table 1). Of note, no F. gigantica sample was misidentified as F. hepatica and vice versa.

Table 1. Identification of 78 adult Fasciola spp. samples obtained from Nigeria and Switzerland by MALDI-TOF MS, using a newly developed in-house database. LSV, log-score value.

\begin{tabular}{ccccc}
\hline Species & Number of Samples & \multicolumn{2}{c}{ Identification } & LSV Range \\
\hline & & LSV $\geq 1.70$ & LSV $\geq 2.00$ & \\
Fasciola gigantica & 75 & $74(98.7 \%)$ & $31(41.3 \%)$ & $1.73-2.13$ \\
Fasciola hepatica & 3 & $3(100 \%)$ & $3(100 \%)$ & $2.15-2.23$ \\
\hline
\end{tabular}




\section{Discussion}

The purpose of this study was to determine whether MALDI-TOF MS can be utilized as a diagnostic tool for the identification and differentiation of adult Fasciola species. Our results show that MSPs created from repetitive measurements of eight adult Fasciola specimens, utilized as part of a helminth-specific in-house database, allowed for an unambiguous identification of 78 additional Fasciola samples. Indeed, 77 of 78 specimens were correctly identified, and there was no misidentification at the species level. A deeper investigation using principal component analysis and discriminant analysis for comparative spectra visualization confirmed species-specific differences that enable accurate diagnosis. In the case of the single specimen that was not correctly identified, we had already noted a slightly different morphological aspect on macroscopic inspection, and no meaningful protein spectra of sufficient quality were obtained by MALDI-TOF MS, which probably indicates previous degradation of the proteins.

MALDI-TOF MS has become a standard diagnostic tool for bacteria $[27,28]$ in the microbiological laboratory and is now increasingly being utilized for identification of yeasts and filamentous fungi as well as mycobacteria. In research settings, entomological studies have also employed MALDI-TOF for identification of ticks [23], mosquitoes [21,29], fleas [30], and lice [31]. Yet, data on application of MALDI-TOF MS for parasites, particularly helminths, are scarce. A recent systematic review identified only five published studies pertaining to diagnostic helminthology [24], using MALDI-TOF MS specifically for Ascaris spp., cyathostomin helminths, Dirofilaria spp. and Trichinella spp. Recently, two studies described the application of MALDI-TOF MS for identification of helminths; one focusing on Trichinella spp. in France [32] and the other on Anisakis spp. in Italy [33]. Using different cestode, nematode and trematode samples, our group has also generated species-specific MSPs and developed an in-house database for helminth identification, which will prospectively be validated on well characterized clinical samples [34]. It is important to note that the protocols used for protein extraction and MALDI-TOF spectra acquisition varied slightly between studies, and the development of one standardized approach will be an important feature to generate (i) accurate databases; and (ii) specific and reproducible results across different laboratories in future studies.

Interestingly, while creating the MSPs for the in-house database, it was observed that two out of seven F. gigantica samples used (i.e., isolates no. FGN23 and FGN24) clustered together in a group with slightly different spectral patterns than the other five F. gigantica samples. Such observed intra-species differences in the mass spectra profiles could be explained by a minor genetic variation (e.g., a non-synonymous mutation, which could affect the protein profile). In this context, it is important to mention that several studies have reported the existence of an intermediate "hybrid" species of Fasciola, which can only be discriminated by specific molecular methods [34]. Liu and colleagues [35] studied the sequence data of protein-encoding genes and showed that the intermediate form of Fasciola is more closely related to F. gigantica than F. hepatica. Similar studies from sub-Saharan Africa have suggested that the epidemiology of fascioliasis in this part of the world may be more complex than previously thought, and that $F$. gigantica is not the only species occurring [36]. "Hybrid" Fasciola spp. have also been reported from Chad [37], and these might also occur in Nigeria, where the F. gigantica samples for the current MALDI-TOF MS were obtained. Since "hybrid" species cannot be accurately identified by amplification of the COX1 as performed here, further molecular investigations pertaining to the genomics (e.g., sequencing of the internal transcribed spacer (ITS) region as reported by Evack and colleagues [37]) of these samples will be interesting. Indeed, while we aligned and comparatively analyzed the obtained COX1 sequences, we were unable to identify specific variable sites, which would have allowed to accurately identify "hybrid" isolates.

Our study is limited by the small sample size for F. hepatica (i.e., only four adult worms), analysis of samples stemming from only two different geographical areas (i.e., Nigeria and Switzerland) and the use of samples preserved in different media (70\% ethanol and $0.7 \% \mathrm{NaCl}$ for $F$. gigantica and F. hepatica, respectively). It is important to mention that 
potential effects of different preservation media used were not thoroughly investigated in this proof-of-concept study. Another limitation relates to the hosts, as we only analyzed Fasciola spp. stemming from animals and further research is needed to confirm that our developed in-house database would also reliably identify flukes from other hosts, e.g., specimens extracted from human bile ducts. It should also be noted that more than half of all F. gigantica samples were identified with LSVs $<2.0$, which would only relate to a genus-specific, but not to a species-specific identification if thresholds for bacteria were used. However, MALDI-TOF MS did not misidentify any of the two Fasciola species. For the further development and improvement of a helminth-specific MALDI-TOF database, a more comprehensive investigation with specimens from other geographical settings in Africa, Asia, Europe and the Americas and samples originating from different hosts would be desirable. Additionally, while we only analyzed adult flukes, future studies should also consider Fasciola eggs and different larval stages to obtain a more accurate taxonomic typing and to further improve the species identification via MALDI-TOF MS. Indeed, a direct MALDI-TOF-based identification of Fasciola eggs in stool samples of human and veterinary origin would represent a major achievement to improve the diagnosis of fascioliasis, including "hybrid" species, in many laboratories. Pending further research on the effect of different storage solutions on the resulting protein spectra, this method might also be employed as a suitable alternative to PCR for a diagnostic identification of helminths after storage for many years (e.g., historical parasite collections in research laboratories).

\section{Conclusions}

We conclude that MALDI-TOF MS is a promising tool for rapid and reliable identification of adult Fasciola and potentially other food-borne trematode species. It is important to note that the creation and validation of a specific in-house database is necessary for identification of taxonomic groups (e.g., food-borne trematodes) that are not covered by commercial databases. To our knowledge, this study is the first to employ MALDI-TOF for differentiation of the causative agents of fascioliasis.

Author Contributions: Conceptualization, I.S., E.O.N., S.P. and S.L.B.; methodology, I.S., L.M., E.O.N and S.L.B.; contribution of samples, E.O.N., M.I.A., S.T.Y., J.K. and S.P.; investigation and formal analysis, I.S., L.M. and E.O.N.; writing—original draft preparation, I.S.; writing—review and editing, I.S., E.O.N., J.R., J.U., S.P. and S.L.B. All authors have read and agreed to the published version of the manuscript.

Funding: E.O.N. was supported by the Cooperation Visits Programme of The World Academy of Sciences and Deutsche Forschungsgemeinschaft (TWAS-DFG). We acknowledge support by the Deutsche Forschungsgemeinschaft (DFG, German Research Foundation) and Saarland University within the funding programme Open Access Publishing.

Institutional Review Board Statement: Written permission was obtained from the management board of the Kubwa abattoir (Abuja, Nigeria) for collection and subsequent analyses of the fluke samples from slaughtered cattle (reference no.: FCT/ARD/TRN/004, 17 October 2019). No approval was required for analysis of the Swiss samples.

Informed Consent Statement: Not applicable.

Data Availability Statement: The data presented in this study are available on request from the corresponding author.

Acknowledgments: We are grateful to Maureen Feucherolles from the Luxembourg Institute of Science and Technology (LIST) for assistance during statistical analysis. We are grateful to Anna Stuermann from the Swiss Tropical and Public Health Institute for isolating the F. hepatica samples from animal livers in the Swiss component of the study.

Conflicts of Interest: The authors declare no conflict of interest.

\section{References}

1. Keiser, J.; Utzinger, J. Food-borne trematodiases. Clin. Microbiol. Rev. 2009, 22, 466-483. [CrossRef] [PubMed]

2. Amer, S.; ElKhatam, A.; Zidan, S.; Feng, Y.; Xiao, L. Identity of Fasciola spp. in sheep in Egypt. Parasit. Vectors 2016, 9, 623. [CrossRef] [PubMed] 
3. Calvani, N.E.D.; Ichikawa-Seki, M.; Bush, R.D.; Khounsy, S.; Šlapeta, J. Which species is in the faeces at a time of global livestock movements: Single nucleotide polymorphism genotyping assays for the differentiation of Fasciola spp. Int. J. Parasitol. 2020, 50, 91-101. [CrossRef] [PubMed]

4. Takeuchi-Storm, N.; Denwood, M.; Petersen, H.H.; Enemark, H.L.; Stensgaard, A.-S.; Sengupta, M.E.; Beesley, N.J.; Hodgkinson, J.; Williams, D.; Thamsborg, S.M. Patterns of Fasciola hepatica infection in Danish dairy cattle: Implications for on-farm control of the parasite based on different diagnostic methods. Parasit. Vectors 2018, 11, 674. [CrossRef]

5. Perrodin, S.; Walti, L.; Gottstein, B.; Kim-Fuchs, C.; Candinas, D.; Banz, V. Fasciola hepatica in a country of low incidence: A tricky diagnosis. Hepatobiliary Surg. Nutr. 2019, 8, 597-603. [CrossRef]

6. Mucheka, V.T.; Lamb, J.M.; Pfukenyi, D.M.; Mukaratirwa, S. DNA sequence analyses reveal co-occurrence of novel haplotypes of Fasciola gigantica with F. hepatica in South Africa and Zimbabwe. Vet. Parasitol. 2015, 214, 144-151. [CrossRef]

7. Chougar, L.; Mas-Coma, S.; Artigas, P.; Harhoura, K.; Aissi, M.; Agramunt, V.H.; Bargues, M.D. Genetically "pure" Fasciola gigantica discovered in Algeria: DNA multimarker characterization, trans-Saharan introduction from a Sahel origin and spreading risk into north-western Maghreb countries. Transbound. Emerg. Dis. 2020. [CrossRef]

8. Mas-Coma, S.; Valero, M.A.; Bargues, M.D. Fasciola, lymnaeids and human fascioliasis, with a global overview on disease transmission, epidemiology, evolutionary genetics, molecular epidemiology and control. Adv. Parasitol. 2009, 69, 41-146. [CrossRef]

9. Jean-Richard, V.; Crump, L.; Abicho, A.A.; Naré, N.B.; Greter, H.; Hattendorf, J.; Schelling, E.; Zinsstag, J. Prevalence of Fasciola gigantica infection in slaughtered animals in south-eastern Lake Chad area in relation to husbandry practices and seasonal water levels. BMC Vet. Res. 2014, 10, 81. [CrossRef]

10. Nguyen, N.T.; Le, T.C.; Vo, M.D.C.; Van Cao, H.; Nguyen, L.T.; Ho, K.T.; Nguyen, Q.N.; Tran, V.Q.; Matsumoto, Y. High prevalence of cattle fascioliasis in coastal areas of Thua Thien Hue province, Vietnam. J. Vet. Med. Sci. 2017, 79, 1035-1042. [CrossRef]

11. Chikowore, T.J.; Zishiri, O.T.; Mukaratirwa, S. Phylogenetic analysis of Fasciola spp. isolated from slaughtered cattle in KwaZulu-Natal and Mpumalanga provinces of South Africa based on the cytochrome c oxidase subunit I mitochondrial marker. Onderstepoort J. Vet. Res. 2019, 86, e1-e11. [CrossRef] [PubMed]

12. Bargues, M.D.; Vigo, M.; Horak, P.; Dvorak, J.; Patzner, R.A.; Pointier, J.P.; Jackiewicz, M.; Meier-Brook, C.; Mas-Coma, S. European Lymnaeidae (Mollusca: Gastropoda), intermediate hosts of trematodiases, based on nuclear ribosomal DNA ITS-2 sequences. Infect. Genet. Evol. 2001, 1, 85-107. [CrossRef]

13. Walker, S.M.; Makundi, A.E.; Namuba, F.V.; Kassuku, A.A.; Keyyu, J.; Hoey, E.M.; Prödohl, P.; Stothard, J.R.; Trudgett, A. The distribution of Fasciola hepatica and Fasciola gigantica within southern Tanzania-constraints associated with the intermediate host. Parasitology 2008, 135, 495-503. [CrossRef] [PubMed]

14. Pointier, J.-P.; Coustau, C.; Rondelaud, D.; Theron, A. Pseudosuccinea columella (Say 1817) (Gastropoda, Lymnaeidae), snail host of Fasciola hepatica: First record for France in the wild. Parasitol. Res. 2007, 101, 1389-1392. [CrossRef]

15. Cwiklinski, K.; Dalton, J.P.; Dufresne, P.J.; La Course, J.; Williams, D.J.; Hodgkinson, J.; Paterson, S. The Fasciola hepatica genome: Gene duplication and polymorphism reveals adaptation to the host environment and the capacity for rapid evolution. Genome Biol. 2015, 16, 71. [CrossRef]

16. Sumruayphol, S.; Siribat, P.; Dujardin, J.-P.; Dujardin, S.; Komalamisra, C.; Thaenkham, U. Fasciola gigantica, F. hepatica and Fasciola intermediate forms: Geometric morphometrics and an artificial neural network to help morphological identification. PeerJ 2020, 8, e8597. [CrossRef]

17. Ai, L.; Chen, M.-X.; Alasaad, S.; Elsheikha, H.M.; Li, J.; Li, H.-L.; Lin, R.-Q.; Zou, F.-C.; Zhu, X.-Q.; Chen, J.-X. Genetic characterization, species differentiation and detection of Fasciola spp. by molecular approaches. Parasit. Vectors 2011, 4, 101. [CrossRef]

18. Chabriere, E.; Bassène, H.; Drancourt, M.; Sokhna, C. MALDI-TOF MS and point of care are disruptive diagnostic tools in Africa. New Microbes New Infect 2018, 26, S83-S88. [CrossRef]

19. Clark, A.E.; Kaleta, E.J.; Arora, A.; Wolk, D.M. Matrix-assisted laser desorption ionization-time of flight mass spectrometry: A fundamental shift in the routine practice of clinical microbiology. Clin. Microbiol. Rev. 2013, 26, 547-603. [CrossRef]

20. Angeletti, S. Matrix assisted laser desorption time of flight mass spectrometry (MALDI-TOF MS) in clinical microbiology. J. Microbiol. Methods 2017, 138, 20-29. [CrossRef]

21. Laroche, M.; Almeras, L.; Pecchi, E.; Bechah, Y.; Raoult, D.; Viola, A.; Parola, P. MALDI-TOF MS as an innovative tool for detection of Plasmodium parasites in Anopheles mosquitoes. Malar. J. 2017, 16, 5. [CrossRef] [PubMed]

22. Nebbak, A.; Willcox, A.C.; Bitam, I.; Raoult, D.; Parola, P.; Almeras, L. Standardization of sample homogenization for mosquito identification using an innovative proteomic tool based on protein profiling. Proteomics 2016, 16, 3148-3160. [CrossRef] [PubMed]

23. Diarra, A.Z.; Almeras, L.; Laroche, M.; Berenger, J.-M.; Koné, A.K.; Bocoum, Z.; Dabo, A.; Doumbo, O.; Raoult, D.; Parola, P. Molecular and MALDI-TOF identification of ticks and tick-associated bacteria in Mali. PLoS Negl. Trop. Dis. 2017, 11, e0005762. [CrossRef] [PubMed]

24. Feucherolles, M.; Poppert, S.; Utzinger, J.; Becker, S.L. MALDI-TOF mass spectrometry as a diagnostic tool in human and veterinary helminthology: A systematic review. Parasit. Vectors 2019, 12, 245. [CrossRef] [PubMed]

25. Hall, T.A. BioEdit: A user-friendly biological sequence alignment editor and analysis program for Windows 95/98/NT. In Proceedings of the Nucleic Acids Symposium Series; Information Retrieval Ltd.: London, UK, 1999; Volume 41, pp. 95-98. 
26. National Center for Biotechnology Information Nucleotide BLAST: Search Nucleotide Databases Using A Nucleotide Query. Available online: https://blast.ncbi.nlm.nih.gov/Blast.cgi?PROGRAM=blastn\&PAGE_TYPE=BlastSearch\&LINK_LOC= blasthome (accessed on 18 November 2020).

27. Welker, M. Proteomics for routine identification of microorganisms. Proteomics 2011, 11, 3143-3153. [CrossRef]

28. Shannon, S.; Kronemann, D.; Patel, R.; Schuetz, A.N. Routine use of MALDI-TOF MS for anaerobic bacterial identification in clinical microbiology. Anaerobe 2018, 54, 191-196. [CrossRef]

29. Nebbak, A.; Almeras, L. Identification of Aedes mosquitoes by MALDI-TOF MS biotyping using protein signatures from larval and pupal exuviae. Parasit Vectors 2020, 13, 161. [CrossRef]

30. Nebbak, A.; El Hamzaoui, B.; Berenger, J.-M.; Bitam, I.; Raoult, D.; Almeras, L.; Parola, P. Comparative analysis of storage conditions and homogenization methods for tick and flea species for identification by MALDI-TOF MS. Med. Vet. Entomol. 2017, 31, 438-448. [CrossRef]

31. Ouarti, B.; Laroche, M.; Righi, S.; Meguini, M.N.; Benakhla, A.; Raoult, D.; Parola, P. Development of MALDI-TOF mass spectrometry for the identification of lice isolated from farm animals. Parasite 2020, 27, 28. [CrossRef]

32. Karadjian, G.; Bilska-Zając, E.; Bahn, P.; Py, J.-S.; Johne, A.; Gassilloud, B.; Różycki, M.; Cencek, T.; Mayer-Scholl, A.; Vallée, I. Species identification of Trichinella originated from various host and different geographical location by MALDI-TOF. Exp. Parasitol. 2020, 213, 107890. [CrossRef]

33. Marzano, V.; Pane, S.; Foglietta, G.; Levi Mortera, S.; Vernocchi, P.; Onetti Muda, A.; Putignani, L. Mass spectrometry-based proteomic analysis of Anisakis spp.: A preliminary study towards a new diagnostic tool. Genes 2020, 11, 693. [CrossRef] [PubMed]

34. Sy, I.; Wendel, T.; Feucherolles, M.; Nimmesgern, A.; Stuermann, A.; Endriss, Y.; Utzinger, J.; Poppert, S.; Becker, S.L. Application of MALDI-TOF MS for identification of helminths in clinical samples. Abstract no. 6730. In Proceedings of the European Congress of Clinical Microbiology and Infectious Diseases, Paris, France, 18-21 April 2020; Cancelled Due to the COVID-19 Pandemic. p. 3232 .

35. Nguyen, T.B.N.; De, N.V.; Nguyen, T.K.L.; Quang, H.H.; Doan, H.T.T.; Agatsuma, T.; Le, T.H. Distribution status of hybrid types in large liver flukes, Fasciola species (Digenea: Fasciolidae), from ruminants and humans in Vietnam. Korean J. Parasitol. 2018, 56, 453-461. [CrossRef] [PubMed]

36. Liu, G.-H.; Gasser, R.B.; Young, N.D.; Song, H.-Q.; Ai, L.; Zhu, X.-Q. Complete mitochondrial genomes of the "intermediate form" of Fasciola and Fasciola gigantica, and their comparison with F. hepatica. Parasit. Vectors 2014, 7, 150. [CrossRef] [PubMed]

37. Evack, J.G.; Schmidt, R.S.; Boltryk, S.D.; Voss, T.S.; Batil, A.A.; Ngandolo, B.N.; Greter, H.; Utzinger, J.; Zinsstag, J.; Balmer, O. Molecular confirmation of a Fasciola gigantica $\times$ Fasciola hepatica hybrid in a Chadian bovine. J. Parasitol. 2020, 106, $316-322$. [CrossRef] [PubMed] 